Details of this experimental service will be announced later; but meanwhile it may be mentioned that the frequencies of the following trans. mitters of the General Post Office and of the B.B.C. are maintained at their nominal values to a tolerance better than \pm 1 part in $10^{6}$.

\begin{tabular}{|c|c|c|c|}
\hline $\begin{array}{c}\text { Station's } \\
\text { call sign }\end{array}$ & Nature of service & Location & $\begin{array}{c}\text { Nominal frequency } \\
\text { kc./s. }\end{array}$ \\
\hline GBR & Telegraph & Rugby & 16 \\
GRO & Broadcast & Droitwich & 200 \\
GSB & Broadcast & Skelton & 6,180 \\
GSV & Broadcast & Daventry & 9,510 \\
& Broadcast & Daventry & 17,810 \\
\hline
\end{tabular}

The B.B.C. medium-wave transmitters, with the exception of that on $583 \mathrm{kc} . / \mathrm{s}$. $(514 \mathrm{~m}$.), are also maintained on their nominal frequencies to a tolerance of approximately \pm 1 part in $10^{6}$.

${ }^{1}$ National Bureau of Standards (U.S.A.). Letter Circular LC886, January $30,1948$.

2 Wireless World, 53, 439 (Nov., 1947).

\section{ELECTRIC CONDUCTIVITY OF ROCKS AT HIGH TEMPERATURES}

$\mathrm{H}$ - P. COSTER has described the results of measurements of the electric conductivities of various rocks (Mon. Not. Roy. Astro. Soc., Geophys. Supp., 5, No. 6, January 1948). The conductivity was determined on specimens of rock cut in the shape of a cylinder, by measuring the resistance between the electrodes in a D.C. bridge circuit; the current in the bridge was reversed after balancing, with the object of detecting potentials due to polarization of the rock by electrolysis. Although a small effect was usually found, its influence on the resistance was never more than a few per cent. The conductivities determined for different rocks showed that they could be classified as semi-conductors, and in many semi-conductors the relation between the conductivity and the absolute temperature $T$ is given by

$\sigma=A_{1} \exp \left(-\varepsilon_{1} / k T\right)+A_{2} \exp \left(-\varepsilon_{2} / k T\right)+\ldots$,

where $k$ is Boltzmann's constant, $\varepsilon_{1}, \varepsilon_{2}$, etc., are the activation energies of the various processes to which the conductivity is due, and $A_{1}, A_{2}$, etc., are constants.

The results of the experiments are shown graphically, $\log _{10} \sigma$ being plotted against $10^{3} / T$, for eight different rocks-all Scottish-which Prof. C. E. Tilley placed at the disposal of the author. The curves show that the conductivity of most of the rocks is due to at least two mechanisms : one with an activation energy of about 0.7 volt and the other with an activation energy of the order of 2 volts, the latter appearing at higher temperatures; experiments on the nature of the conductivity suggest that in this case it is caused by moving ions.

The theoretical implications of the results with regard to the conductivity in the interior of the earth are discussed, and the following suggestions are made. At a depth of a few kilometres where the rocks are probably dry, the conductivity is lower than it is nearer the surface; but as greater depths are reached, where the temperature is higher, the conductivity should start to increase. Assuming that pressure has no effect on the conductivity, a value of $0.01 \mathrm{ohm}^{-1} \mathrm{~cm}^{-1}$ should be reached at a temperature of $1,200-1,400^{\circ} \mathrm{C}$., possibly at a depth of $160 \mathrm{~km}$. The effect of pressure is, however, a serious source of uncertainty in the investigation; but using the temperatures and pressures given by Bullard and Bullen, respectively, Coster has compiled a table which shows the variation of temperature, pressure and conductivity with depth, though the relation cannot be regarded as anything more than an approximation. At depths greater than $200 \mathrm{~km}$. the increase in conductivity is rather small, because the effect of increase in temperature is almost compensated by that of increase in pressure. The figures, though giving only a rough idea of the varia. tion of conductivity with depth, nevertheless can account for the increase of conductivity with depth found by Chapman and Price.

\section{MEXICAN VILLAGE LIFE}

$T$ HE monograph under notice*, the sixth of a series dealing with the sociology of Latin America, returns to the Mexican Highlands and to the Tarascan pueblo of Tzintzunzan. This village, which at the time of the Spanish conquest was the capital of an empire second only to the Aztec in size, has now become a sleepy Mestizo community of 1,500 souls which is "probably typical of a vast number of small rural Mexican pueblos". "Empire's Children" is a very readable account of this engaging and happy-go-lucky community of potters and farmers where "the great majority of the villagers get down to work only with a tremendous exertion of will power and stop on the slightest pretext", and where "Russia, Communism, the United States and Protestantism are conveniently lumped together as enemies of the inalienable rights of man".

The author's purpose is to convey a general and comprehensive impression of the social and economic life of these people, and he has been more than usually successful in achieving this. The reader is neither overwhelmed with masses of unnecessary detail nor mystified by technical anthropological jargon, and yet practically every aspect of village life is covered-its history and prehistory, its domestic and its material culture, its trade and commerce and its social, political and religious institutions. His summing up and conclusions are not so impressive, probably because of his short acquaintance with the country (two years) and possibly because although he admits the essential unity of the Mestizo culture, he remains too preoccupied in distinguishing the Spanish and Tarascan traits which contribute to its 'total con. figuration'. However, this summary only takes up eight pages at the end of the monograph and is probably inserted for the benefit of the Mexican Government rather than for the general reader.

It is to be hoped that this preliminary survey will be succeeded by detailed studies of the more striking aspects of rural Mexican society, in particular, of the part played by the Church, a role so vital that Foster considers it the "socio-spiritual focal point of the culture".

G. I. JoNES

* Empire's Children. The People of Tzintzunzan. By George M. Foster assisted by Gabriel Ospina. Pp. $v+297+16$ plates. (Pub No. 6, Institute of Social Anthropology, Smithsonian Institution, Washington, D.C.)

See also Nature, 161, 697 (1948) for notice of Pub. 2-5 of this series. 